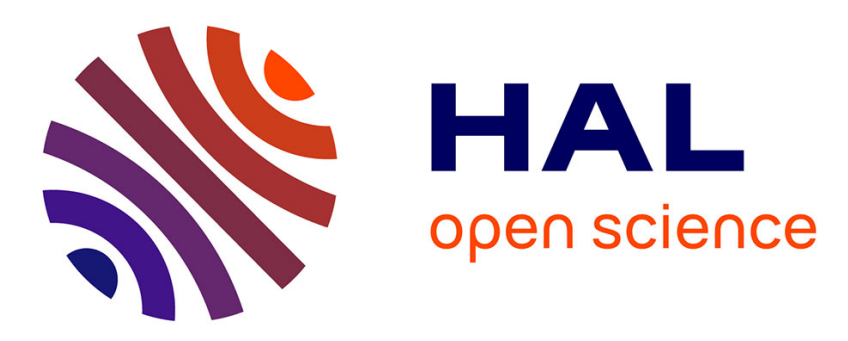

\title{
Analogies Between Social Interaction Models and Supply Chains
}

\author{
Laurent Navoret, Richard Bon, Pierre Degond, Jacques Gautrais, David \\ Sanchez, Guy Theraulaz
}

\section{To cite this version:}

Laurent Navoret, Richard Bon, Pierre Degond, Jacques Gautrais, David Sanchez, et al.. Analogies Between Social Interaction Models and Supply Chains. European Consortium for Mathematics in Industry (ECMI) conference, 2008, London, United Kingdom. hal-01872081

\section{HAL Id: hal-01872081 https://hal.science/hal-01872081}

Submitted on 11 Sep 2018

HAL is a multi-disciplinary open access archive for the deposit and dissemination of scientific research documents, whether they are published or not. The documents may come from teaching and research institutions in France or abroad, or from public or private research centers.
L'archive ouverte pluridisciplinaire $\mathbf{H A L}$, est destinée au dépôt et à la diffusion de documents scientifiques de niveau recherche, publiés ou non, émanant des établissements d'enseignement et de recherche français ou étrangers, des laboratoires publics ou privés. 


\section{Analogies between social interactions models and supply chains}

Laurent Navoret ${ }^{1}$, Richard Bon ${ }^{2}$, Pierre Degond ${ }^{3}$, Jacques Gautrais ${ }^{4}$, David Sanchez ${ }^{5}$, and Guy Theraulaz ${ }^{6}$

1 Institut de Mathématiques de Toulouse, Université Paul Sabatier, 118 route de Narbonne, 31062 Toulouse Cedex 9 France,

laurent.navoret@math. univ-toulouse.fr

2 Centre de Recherche en Cognition Animale, Université Paul Sabatier, 118 route de Narbonne, 31062 Toulouse Cedex 9 France, rbon@cict.fr

3 Institut de Mathématiques de Toulouse, pierre.degond@math.univ-toulouse.fr

4 Centre de Recherche en Cognition Animale, gautrais@cict.fr

5 Institut de Mathématiques de Toulouse, david.sanchez@math.univ-toulouse.fr

${ }^{6}$ Centre de Recherche en Cognition Animale, theraula@cict.fr

Congestion is a major issue in the modeling of both animal aggregation and supply chains. Indeed, both systems face obvious physical limits : capacities of machine in supply chains [1] and non-overlapping constraints between individuals in social groups. As a paradigm of crowding and social group movement, we are interested in sheep herds. The behaviour of this gregarious animal is experimentally studied [5]. Let us focus on the displacement period of a sheep herd, where all animals move with the same speed.

A macroscopic model for herds including speed and congestion constraints is derived from an individual based model. In order to enlight the congestion part in the dynamics, a singular limit of this macroscopic model is taken and leads to two phases in our herd : a congested and a non-congested one. We finally analyse the spatial transition between these two phases. Such a study of the congestion in self organized systems could be translated to supply chains context. 


\section{Derivation of a macroscopic model with speed and congestion constraints}

\subsection{Microscopic model}

The following microscopic model aims to describe the interactions of $N$ particles labeled by $k \in\{1, . ., N\}$ and with position $\mathbf{X}_{k} \in \mathbb{R}^{2}$ with two main contraints.

The first constraint consists in supposing that all the particles have the same magnitude of velocity, here equal to 1 . In first approximation, this assumption is satisfied by the sheeps in a moving herd [5] (or schools of fish [4]). Thus the velocity of the $k$-th particle is given by $\boldsymbol{\omega}_{k}$, where $\boldsymbol{\omega}_{k} \in \mathbb{S}^{1}=\left\{\boldsymbol{\omega} \in \mathbb{R}^{2},|\boldsymbol{\omega}|=1\right\}$ is an unitary vector. Therefore the time derivative of $\boldsymbol{\omega}_{k}$ is orthogonal to $\boldsymbol{\omega}_{k}$. The second constraint is the congestion one. The particles are supposed to have a finite volume (equal to $\pi d^{2}$ ) and can not overlap, hence the existence of a maximal density $\varrho^{*}$. So the repulsive interaction has to be singular so as to prevent the density from exceeding $\varrho^{*}$.

We propose here a simple continuous model for the evolution of positions and velocities via attractive-repulsive binary interactions :

$$
\begin{aligned}
\frac{\mathrm{d} \mathbf{X}_{k}}{\mathrm{~d} t} & =\boldsymbol{\omega}_{k}, \\
\frac{\mathrm{d} \boldsymbol{\omega}_{k}}{\mathrm{~d} t} & =\nu_{k}^{a}\left(\mathrm{Id}-\boldsymbol{\omega}_{k} \otimes \boldsymbol{\omega}_{k}\right) \boldsymbol{\xi}_{k}^{a}-\nu_{k}^{r}\left(\mathrm{Id}-\boldsymbol{\omega}_{k} \otimes \boldsymbol{\omega}_{k}\right) \boldsymbol{\xi}_{k}^{r},
\end{aligned}
$$

where $\boldsymbol{\xi}_{k}^{a, r}$ are the attractive-repulsive forces, $\nu_{a, r}$ their respective interaction frequencies. The matrix $\left(\mathrm{Id}-\boldsymbol{\omega}_{k} \otimes \boldsymbol{\omega}_{k}\right)$ is the orthogonal projector on the orthogonal direction to $\boldsymbol{\omega}_{k}$ and enables to satisfy the speed constraint. The attractive force is chosen to drive the particles to the centre of mass inside an interaction disc of radius $R_{a}$, while the repulsive force is chosen to drive them to the opposite direction of the centre of mass inside an interaction disc of radius $R_{r}$ (lower than $R_{a}$ ):

$$
\boldsymbol{\xi}_{k}^{a}=\frac{\sum_{j,\left|\mathbf{X}_{j}-\mathbf{X}_{k}\right| \leq R_{a}} \mathbf{X}_{j}-\mathbf{X}_{k}}{\sum_{j,\left|\mathbf{X}_{j}-\mathbf{X}_{k}\right| \leq R_{a}} 1}, \quad \boldsymbol{\xi}_{k}^{r}=\frac{\sum_{j,\left|\mathbf{X}_{j}-\mathbf{X}_{k}\right| \leq R_{r}} \mathbf{X}_{j}-\mathbf{X}_{k}}{\sum_{j,\left|\mathbf{X}_{j}-\mathbf{X}_{k}\right| \leq R_{r}} 1} .
$$

The attractive interaction is a constant $\nu_{k}^{a}=\nu_{a}$ and $\nu_{r}$ is taken so as to satisfy the congestion constraint :

$\nu_{k}^{r}=\nu_{r}\left(\frac{\pi d^{2} \sum_{j,\left|\mathbf{X}_{j}-\mathbf{X}_{k}\right| \leq R_{r}} 1}{\pi R_{r}^{2}}\right), \quad \nu_{r}(\varrho)=\varrho p^{\prime}(\varrho), \quad p(\varrho)=\left(\frac{1}{\varrho^{*}}-\frac{1}{\varrho}\right)^{-k}$.

Note that $p(\varrho)$ tends to $+\infty$ when $\varrho$ goes to $\varrho^{*}$. The form of the function $\nu_{r}$ is explicitly given only for the convenience of the following study. 


\subsection{Kinetic model, hydrodynamic rescaling and macroscopic model}

Mean-field limit : $N \rightarrow+\infty$

To describe the dynamics of a large number of particles, it is usual in mathematical physics to introduce a distribution function $f(\mathbf{x}, \mathbf{v}, t)$ defined on the phase space : $f(\mathbf{x}, \mathbf{v}, t) \mathrm{d} \mathbf{x} \mathrm{d} \mathbf{v}$ is the number of particles in the volume $[\mathbf{x}, \mathbf{x}+\mathrm{d} \mathbf{x}] \times[\mathbf{v}, \mathbf{v}+\mathrm{d} \mathbf{v}]$. From the equations satisfied by the empirical distribution $f^{N}(\mathbf{x}, \boldsymbol{\omega}, t)=\frac{1}{N} \sum_{k=1}^{N} \delta\left(\mathbf{x}-\mathbf{X}_{k}(t)\right) \delta\left(\boldsymbol{\omega}, \boldsymbol{\omega}_{k}(t)\right)$, we can formally derive the limit equation satified by $f=\lim f^{N}$ as the number of particles tends to $+\infty$ :

$$
\begin{aligned}
& \partial_{t} f+\boldsymbol{\omega} \cdot \nabla_{\mathbf{x}} f+\nabla_{\boldsymbol{\omega}} \cdot\left(\left(\mathbf{F}_{a}-\mathbf{F}_{r}\right) f\right)=0, \\
& \mathbf{F}_{a, r}(x, \boldsymbol{\omega}, t)=\nu_{a, r}(\mathrm{Id}-\boldsymbol{\omega} \otimes \boldsymbol{\omega}) \boldsymbol{\xi}_{a, r}, \\
& \boldsymbol{\xi}_{a, r}(x, \omega, t)=\frac{\int K_{a, r}(\mathbf{y}-\mathbf{x})(\mathbf{y}-\mathbf{x}) \varrho(\mathbf{y}, t) d \mathbf{y}}{\int K_{a, r}(\mathbf{y}-\mathbf{x}) \varrho(\mathbf{y}, t) d \mathbf{y}}, \\
& \nu_{r}=\nu_{r}\left(\frac{\int K_{r}(\mathbf{y}-\mathbf{x}) \varrho^{N}(\mathbf{y}, t) d \mathbf{y}}{\alpha \int K_{r}(\mathbf{y}-\mathbf{x}) d \mathbf{y}}\right),
\end{aligned}
$$

where $\varrho(\mathbf{x}, \mathbf{v}, t)=\int f(\mathbf{x}, \mathbf{v}, t) d \mathbf{v}$ is the density and $K_{a, r}$ are the characteristic functions of the discs of radius $R_{a}$ and $R_{r}$.

\section{Hydrodynamic scaling}

To determine now the large time and space dynamics, we perform an hydrodynamic scaling. Let us introduce the new time and space variables : $\widetilde{\mathbf{x}}=\eta \mathbf{x}$, $\widetilde{t}=\eta t$, with $\eta<<1$. With this rescaling, the repulsive terms become local $: \nu_{r}^{\eta}(\mathbf{x})=\nu_{r}(\varrho(\mathbf{x}))+o(\eta), \xi_{r}^{\eta}(\mathbf{x}, \boldsymbol{\omega}, t)=\frac{R_{r}^{2}}{4} \nabla_{\mathbf{x}} \varrho^{\eta}(\mathbf{x}, t) / \varrho^{\eta}(\mathbf{x}, t)+o(\eta)$. As regards the attractive terms, we suppose that it remains non local as $\eta$ tends to 0 and weaker than the repulsive force : the scaled attractive kernel $K_{a}^{\eta}$ and the scaled interaction frequency $\nu_{a}^{\eta}$ satisfy $K_{a}^{\eta}(\mathbf{z})=K_{a}(\eta \mathbf{z}), \nu_{a}^{\eta}=\eta^{2} \nu_{a}$. Under all these model assumptions, the limit distribution function $f$ we obtain as $\eta$ tends to 0 in the new variables satisfies the system

$$
\begin{aligned}
& \partial_{t} f+\boldsymbol{\omega} \cdot \nabla_{\mathbf{x}} f+\nabla_{\boldsymbol{\omega}} \cdot\left(\left(\mathbf{F}_{a}-\mathbf{F}_{r}\right) f\right)=0, \\
& \mathbf{F}_{a}(x, \omega, t)=\nu_{a}(\operatorname{Id}-\boldsymbol{\omega} \otimes \boldsymbol{\omega}) \boldsymbol{\xi}_{a}, \boldsymbol{\xi}_{a}(x, t)=\left(\frac{\int K_{a}(|\mathbf{y}-\mathbf{x}|)(\mathbf{y}-\mathbf{x}) \varrho(\mathbf{y}, t) d \mathbf{y}}{\int K_{a}(|\mathbf{y}-\mathbf{x}|) \varrho(\mathbf{y}, t) d \mathbf{y}}\right), \\
& \mathbf{F}_{r}(\mathbf{x}, \boldsymbol{\omega}, t)=\frac{R_{r}^{2}}{4}(\operatorname{Id}-\boldsymbol{\omega} \otimes \boldsymbol{\omega}) \nabla_{\mathbf{x}} p(\varrho(\mathbf{x}, t)) .
\end{aligned}
$$

Macroscopic model

The last step of our derivation of models is to find the equation satisfied by the two first moments of the distribution function $f$ : the density $\varrho=\int f d \boldsymbol{\omega}$ and the momentum $\varrho \boldsymbol{\Omega}=\int f \boldsymbol{\omega} d \boldsymbol{\omega}$. Supposing that $f$ is regular enough and 
tends quickly enough to zero at infinity, then it can be checked that $\varrho$ and $\varrho \Omega$ satisfy

$$
\begin{aligned}
& \partial_{t} \varrho+\nabla_{\mathbf{x}} \cdot \varrho \boldsymbol{\Omega}=0 \\
& \partial_{t} \varrho \boldsymbol{\Omega}+\nabla_{\mathbf{x}} \cdot\left(\int f \boldsymbol{\omega} \otimes \boldsymbol{\omega} d \boldsymbol{\omega}\right)=\int(I d-\boldsymbol{\omega} \otimes \boldsymbol{\omega}) f d \boldsymbol{\omega}\left(\nu_{a} \boldsymbol{\xi}_{a}-\frac{R_{r}^{2}}{4} \nabla_{\mathbf{x}} p(\varrho)\right)(7)
\end{aligned}
$$

where $\boldsymbol{\xi}_{a}$ is always given by (5). Unfortunately this system is not closed. We have to make new assumptions to express in term of the two first moments the quantities where $f$ still appears. Here we assume that $f$ is a monokinetic distribution : $f(\mathbf{x}, \boldsymbol{\omega}, t)=\varrho(\mathbf{x}, t) \delta(\boldsymbol{\omega}, \boldsymbol{\Omega}(x, t))$, with $|\boldsymbol{\Omega}(x, t)|=1$. Finally, we obtain the following macroscopic system

$$
\begin{aligned}
\partial_{t} \varrho+\nabla_{\mathbf{x}} \cdot \varrho \boldsymbol{\Omega} & =0, \\
\partial_{t}(\varrho \boldsymbol{\Omega})+\nabla_{\mathbf{x}} \cdot(\varrho \boldsymbol{\Omega} \otimes \boldsymbol{\Omega}) & =\varrho(I d-\boldsymbol{\Omega} \otimes \boldsymbol{\Omega})\left(\nu_{a} \boldsymbol{\xi}_{a}-\frac{R_{r}^{2}}{4} \nabla_{\mathbf{x}} p(\varrho)\right) .
\end{aligned}
$$

\section{Study of the dilute-congested transition}

These macroscopic equations (8)-(9) combine the congestion contraint embodied by $p$ and the speed constraint embodied by the projection operator $(I d-\Omega \otimes \Omega$ ). It leads to two difficulties : the singularity of the pressure $p$ and the non-conservativity of the equation (9). The first point has already been tackled in a one dimensional traffic jam model [2]. The goal of the following study is the treatment of the conjunction of the two in a 2 dimensional case. Since attractive and repulsive forces operate at different scales, we consider thereafter the attraction term as a source term and focus on the case $\nu_{a}=0$ and $R_{r}<<1$.

\subsection{Asymptotic model}

So as to study the singularity of the pressure, the principle is to enhance it by changing $p$ into $\varepsilon p, \varepsilon<<1\left(R_{r}^{\varepsilon 2}=\varepsilon R_{r}^{2}\right)$. By this way, the pressure term becomes negligible unless the density is near the maximal one. Let us denote by $\left(\varrho^{\varepsilon}, \boldsymbol{\Omega}^{\varepsilon}\right)$ the solution of the $\varepsilon$-system

$$
\begin{aligned}
& \partial_{t} \varrho^{\varepsilon}+\nabla_{x} \cdot \varrho^{\varepsilon} \boldsymbol{\Omega}^{\varepsilon}=0, \\
& \partial_{t} \boldsymbol{\Omega}^{\varepsilon}+\boldsymbol{\Omega}^{\varepsilon} \cdot \nabla_{\mathbf{x}} \boldsymbol{\Omega}^{\varepsilon}+\frac{R_{r}^{2}}{4}(I d-\boldsymbol{\Omega} \otimes \boldsymbol{\Omega}) \varepsilon \nabla_{\mathbf{x}} p\left(\varrho^{\varepsilon}\right)=0,
\end{aligned}
$$

If $\left(\varrho^{\varepsilon}, \boldsymbol{\Omega}^{\varepsilon}\right)$ is a sequence of solutions converging to a solution $(\varrho, \Omega)$ when $\varepsilon$ tends to zero, then the limit $\bar{p}(x)=\lim _{\varepsilon \rightarrow 0} \varepsilon p\left(\varrho^{\varepsilon}(x, t)\right)$ is equal to zero unless $\varrho^{\varepsilon}$ tends to $\varrho^{*}$. We assume that $\bar{p}$ is always finite. Thus, two interacting phases with different dynamics appears at the limit : the phase of maximal density 
$\varrho=\varrho^{*}$, called congested phase, and the phase of density lower than $\varrho^{*}$, called the dilute phase. The limit $(\varrho, \boldsymbol{\Omega})$ fulfills the system

$$
\begin{aligned}
& \partial_{t} \varrho+\nabla_{\mathbf{x}} \cdot \varrho \boldsymbol{\Omega}=0, \\
& \partial_{t} \boldsymbol{\Omega}+\boldsymbol{\Omega} \cdot \nabla_{\mathbf{x}} \boldsymbol{\Omega}+\frac{R_{r}^{2}}{4}(I d-\boldsymbol{\Omega} \otimes \boldsymbol{\Omega}) \nabla_{\mathbf{x}} \bar{p}=0, \\
& \left(\varrho^{*}-\varrho\right) \bar{p}=0,
\end{aligned}
$$

where the last equality expresses the dichotomy $\varrho=\varrho^{*}$ or $\bar{p}=0$.

In the dilute phase, where the density is lower than $\varrho^{*}$, we get a pressureless gaz dynamic model. Let us now investigate the system in the congested phase.

\subsection{In the congested phase}

In the congested phase $\varrho=\varrho^{*}$, the limit of (10)-(11) leads to an incompressible Euler system with speed constraint

$$
\begin{aligned}
& \varrho=\varrho^{*}, \quad \nabla_{\mathbf{x}} \cdot \boldsymbol{\Omega}=0, \\
& \partial_{t} \boldsymbol{\Omega}+\boldsymbol{\Omega} \cdot \nabla_{\mathbf{x}} \boldsymbol{\Omega}+\frac{R_{r}^{2}}{4}(I d-\boldsymbol{\Omega} \otimes \boldsymbol{\Omega}) \nabla_{\mathbf{x}} \bar{p}=0,
\end{aligned}
$$

where the pressure $\bar{p}$ is the Lagrange multiplier of the incompressibility constraint.

The only incompressibility constraint (15) coupled with the speed constraint provide us enlightening ideas of the structure of clusters. Indeed, we can prove that if the vector field $\boldsymbol{\Omega}$ on the sphere $(|\boldsymbol{\Omega}|=1)$ satisfy the incompressibility constraint (15), then $\boldsymbol{\Omega}$ is constant on straight lines and orthogonal to these lines. Concerning the pressure $\bar{p}$, it satisfies an elliptic equation on the congested domain (easily obtained by taking the divergence of the momentum equation (16)).

As a result of these two last remarks, the only knowledge of the velocity $\boldsymbol{\Omega}$ and the pressure $\bar{p}$ on the border of the congested domain would enable us to find out the whole solution inside the congested zone. So given the interface dynamics, the whole problem could be solved.

\subsection{The interface dynamics}

So as to study the interface dynamics, we consider that our problem at the interface reduces to a one dimensional problem in the normal direction to this interface. Let us focus on the Riemann problem : the initial condition is a discontinuity between two constant states on both sides of the interface. The strategy is here to come back to the finite $\varepsilon$-system (10)-(11) and to extract the limit solutions of the Riemann problem as $\varepsilon$ tends to zero with a left or a right state converging to $\varrho^{*}$. 
By introducing $\theta$ with respect to the $x_{1}$ axis and assuming that the problem is uniform with respect to the $x_{2}$ axis, the non-conservative system (10)-(11) can be put for $\theta \in] 0, \pi[$ in the form of the following conservative one

$$
\begin{aligned}
& \partial_{t} \varrho+\partial_{x_{1}}(\varrho \cos (\theta))=0, \\
& \partial_{t} \Psi(\cos (\theta))+\partial_{x_{1}}\left(\phi(\cos (\theta))+\frac{R_{r}^{2}}{4} \varepsilon p(\varrho)\right)=0,
\end{aligned}
$$

where $\Psi(u)=\frac{1}{2} \log \left(\frac{1+u}{1-u}\right)$ and $\phi(u)=\log \left(\frac{1}{\sqrt{1-u^{2}}}\right)$. The new conserved variables are $\varrho$ and $\Psi(\cos (\theta))$.

This system is strictly hyperbolic and its associated fields are in the limit $\varepsilon=0$ genuinely nonlinear. Therefore, classical results [3] provide us the entropic solution of the Riemann problem. For a non-congested left state $\left(\varrho_{\ell}, \theta_{\ell}\right)$ and a congested right state $\left(\varrho^{*}, \theta_{r}, \bar{p}_{r}\right)$, the two main possibilities are given by

- in case of seperating velocities $\cos \left(\theta_{\ell}\right)<\cos \left(\theta_{r}\right)$, vacuum appears between two contact discontinuities and there is an instantaneous declustering (the pressure becomes zero inside the congested domain);

- in case of incoming velocities $\cos \left(\theta_{\ell}\right)<\cos \left(\theta_{r}\right)$, the limit solution consists of one shock and a pressure jump in the congested domain. The new pressure is computable since it is the solution of an explicit non-linear equation.

The detailed study provides us also the interface dynamics in the other cases $\left(\cos \left(\theta_{\ell}\right)=\cos \left(\theta_{r}\right), \varrho^{\ell}=\varrho^{r}=\varrho^{*}\right.$, etc. $)$. It will be displayed in future papers.

\section{Conclusion}

In this paper, new tools for congestion modeling have been presented in the context of sheep herds modeling. We hope that it could be usefully adapted to supply chains modeling. The study of the congested/non-congested transition will be the ground of further challenging simulations taking into account both constraints (constant speed and maximal density).

\section{References}

1. C. Ringhofer D. Armbruster, P. Degond. Continuum models for interacting machines. World Scientific, 2005.

2. M. Delitala M. Rascle F. Berthelin, P. Degond. A model for the formation and the evolution of traffic jams. Arch. Rational Mech. Anal., 187(2):185-220, 2008.

3. R.J. LeVeque. Numerical Methods for Conservation Laws. Birkhäuser, 1992.

4. S. Motsch P. Degond. Continuum limit of self-driven particles with orientation interaction. preprint.

5. M.H. Pillot. Etude et modélisation des déplacements collectifs spontanés chez le mouton mérinos dArles (ovis aries). Master thesis, 2006. 